\title{
Influence de l'élasticité de la paroi sur l'évolution des ondes de pression dans les réseaux de conduites
}

\author{
Jawhar Gargouri ${ }^{1, a}$, Ezzeddine Hadj-Taieb ${ }^{1}$ et Claude Thirriot $^{2}$ \\ 1 Unité de Mécanique des fluides appliquée et modélisation, ENIS, BP W 3038, Sfax, Tunisie \\ 2 Institut de mécanique des fluides, 2 rue Camichel, 31000 Toulouse, France
}

Reçu le 4 novembre 2006, accepté le 3 décembre 2007

\begin{abstract}
Résumé - Dans cette étude, on examine, par simulation numérique, la possibilité de réduire les pressions provoquées par le phénomène du coup de bélier dans un réseau de conduites quasi-rigides en remplaçant l'une des conduites du réseau par une conduite viscoélastique en polymère. Le modèle numérique développé est constitué d'un système de deux équations aux dérivées partielles non-linéaires de type hyperbolique résolu par la méthode des caractéristiques avec mise en mémoire et interpolation. Le calcul au niveau des nœuds est effectué par la résolution d'un système linéaire avec prise en considération des conditions aux limites imposées. La loi de comportement de la conduite viscoélastique est décrite par le modèle de KelvinVoigt. Afin de simplifier les calculs, on se limite à l'élément élastique de ce modèle. Pour les applications, un code de calcul écrit en langage FORTRAN a été élaboré. Les résultats obtenus montrent bien l'effet de l'élasticité des parois sur l'évolution et l'amortissement des ondes de pression dans les réseaux de conduites.
\end{abstract}

Mots clés : Coup de bélier / méthode des caractéristiques / amortissement / célérité / réseaux de conduites / viscoélasticité

\begin{abstract}
Influence of the pipe wall elasticity on the evolution of the pressure waves in the networks. In this paper, the possibility of reducing pressure surges in a quasi-rigid pipe network by replacing one of the pipes of the network by a viscoelastic one is examined. The mathematical model consists of a system of two hyperbolic, partial differential equations which are resolved by the method of characteristics using linear interpolation. The calculation at the network junctions is realised by resolving a linear system of equations including the manner various boundary conditions are introduced into the solution. The behaviour law of the viscoelastic pipe is described by the Kelvin-Voigt model. In order to simplify calculations, only the elastic element of this model is considered. For the applications, a constructed computer program written in FORTRAN language is elaborated. The numerical results show the effect of the pipe wall elasticity on the damping of the pressure waves in the networks.
\end{abstract}

Key words: water hammer / method of characteristics / damping / pressure wave celerity / pipe networks / viscoelasticity

\section{Introduction}

La gestion des ressources hydriques a consisté d'abord et naturellement à s'assurer de la disponibilité de la ressource puis de la qualité des eaux. Ce n'est que récemment que le problème des fuites et du rendement de la distribution s'est posé avec une certaine acuité (Mpesha et al. [1]). Ces fuites sont dues à la corrosion ou à la rupture d'éléments des circuits d'adduction. La rupture des canalisations, phénomène assez fréquent en milieu urbain, s'amorce sur un défaut (qui peut être dû à la corrosion)

\footnotetext{
a Auteur pour correspondance : tis.technique@tunet.tn
}

sous l'effet des contraintes engendrées par les coups de bélier, ou le mouvement des canalisations par la modification de la rigidité des fondations.

Certains matériaux sont quasi unanimement cités comme posant des problèmes : il s'agit de l'acier et de la fonte grise, très cassants. Les canalisations faites de ces matériaux sont donc susceptibles de présenter des casses plus ou moins nombreuses.

Or, le renouvellement de toutes les conduites du réseau pose des problèmes au niveau de l'importance des financements à mettre en œuvre. Il est donc intéressant d'œuvrer dans le sens d'allonger la durée de vie des conduites du réseau. Le principe est simple; il suffit de réduire les 


\section{Nomenclature}

\begin{tabular}{|ll|}
\hline$C$ & Célérité des ondes de pression \\
$D$ & Diamètre de la conduite \\
$E$ & Module de Young \\
$H$ & Charge \\
$J$ & Perte de charge \\
$L$ & Longueur de la conduite \\
$p$ & Pression du fluide \\
$Q$ & Débit \\
$S$ & Section de la conduite \\
$t$ & Temps \\
$x$ & Abscisse \\
$\lambda$ & Coefficient de friction \\
$\rho$ & Densité du fluide \\
\hline
\end{tabular}

sollicitations en service notamment les fluctuations de pression provoquées par le phénomène du coup de bélier.

De nombreux travaux expérimentaux ou numériques ont montré la complexité du phénomène de propagation d'onde dans les systèmes de conduites à caractéristiques multiples (Boulos et al. [2], Samani et Khayatzadeh [3], Streeter et Wylie [4]). Cette complexité se traduit par la difficulté de prévision de la surpression maximale, dont l'amplitude et la localisation dans le temps, semblent, d'après les essais numériques, être aléatoires, ce qui est en contradiction avec le caractère déterministe du phénomène.

Dans cette étude, on examine par simulation numérique la possibilité de réduire les surpressions provoquées par le phénomène du coup de bélier dans un réseau maillé ou ramifié de conduites quasi-rigides (métalliques ou en béton) en remplaçant l'une des conduites du réseau par une conduite viscoélastique en polymère.

En effet, le comportement viscoélastique provoque l'atténuation de toute perturbation libre qu'un matériau peut subir. Les études sur les écoulements transitoires dans les conduites déformables montrent comment cet effet peut être très important dans l'amortissement de la surpression et la réduction de la durée du phénomène de coup de bélier même en présence de faible résistance à l'écoulement. Cette réduction de la durée peut être particulièrement utile en présence des surpressions séquentielles (par exemple dans les stations de pompage et au niveau des vannes à fermeture rapide).

Ghilardi et Paoletti [5] ont étudié l'effet sur le phénomène du coup de bélier d'une conduite viscoélastique supplémentaire montée en série avec une conduite élastique quasi-rigide. Ils ont constaté que les ondes de pression sont clairement amorties par la dissipation d'énergie du comportement visqueux du matériau.

D'autres études ont montré l'effet remarquable de la viscoélasticité de la paroi sur la réponse du système durant le régime transitoire rapide (Güney [6], Gally et al. [7], Suo et Wylie [8]). Les résultats montrent que, outre l'amortissement précité, les pressions, qui dépendent de la configuration et de la complaisance (souplesse) de la structure, atteignent au début du régime transitoire, dû à des fermetures rapides de vannes, des pressions égales à celles obtenues par la théorie classique du coup de bélier dans les conduites élastiques (Bahrar [9]). Ces valeurs, qui dépendent de la célérité des ondes, représentent approximativement des bornes supérieures des fluctuations de pression dans les conduites viscoélastiques et peuvent être utilisées comme une estimation approchée de telles pressions.

L'emploi de plus en plus généralisé des conduites en matériaux viscoélastiques (PVC), ou élastiques nonlinéaires (caoutchouc, polyéthylène) dans les réseaux de conduites de distribution d'eau potable, nous a amené à entreprendre cette étude et à se demander si l'on peut réduire les fluctuations de pressions provoquées par le phénomène de coup de bélier en remplaçant l'une des conduites élastiques quasi-rigides du réseau par une conduite viscoélastique.

Pour répondre à cette question, un modèle numérique, basé sur la méthode des caractéristiques avec mise en mémoire et interpolation, a été développé. Le comportement viscoélastique du matériau a été modélisé suivant la loi de Kelvin-Voigt (Germain [10]). Vue la complexité du calcul des écoulements transitoires dans les réseaux de conduites, l'étude sera restreinte à l'élément élastique de ce modèle.

Nous nous sommes donc limités, dans tout ce qui suit, au modèle des conduites élastiques de module d'Young égal au module d'Young instantané. La résolution numérique est obtenue à l'aide d'un code de calcul, écrit en langage Fortran.

\section{Formulation mathématique}

\section{1 Équations de mouvement}

Dans le cas d'un écoulement unidirectionnel dans une conduite cylindrique de comportement élastique linéaire selon la loi de Hooke de diamètre $D$, les relations de base simplifiées de calcul du phénomène de coup de bélier sont représentées par les équations de continuité et de mouvement qui s'écrivent [4] :

$$
\frac{\partial H}{\partial t}+\frac{C^{2}}{g S} \frac{\partial Q}{\partial x}=0 \text { et } \frac{\partial Q}{\partial t}+g S \frac{\partial H}{\partial x}+g S J=0
$$

où $Q$ est le débit, $H$ est la charge, $S$ est la section de la conduite, $J=(\lambda / D) Q|Q| /\left(2 g S^{2}\right)$ est la perte de charge linéaire par unité de longueur de conduite, $\lambda$ est le coefficient de perte de charge linéaire (Carlier [11]) et $C$ est la célérité des ondes de pression qui est fonction des propriétés physiques du fluide et des caractéristiques mécaniques de la conduite.

Bien entendu, ces relations simplifiées ne seraient valables que sous les hypothèses suivantes :

- Le mouvement axial de la conduite est supposé négligeable devant sa déformation radiale;

- L'écoulement est unidirectionnel;

- La paroi de la conduite est élastique et le coefficient de Poisson est proche de 0,5 , ceci signifie que le volume de la paroi par unité de longueur reste constant. 


\subsection{Célérité des ondes de pression}

Selon Stuckenbruck et al. [12], la célérité des ondes de pression dans les écoulements transitoires en conduites déformables peut s'exprimer par la relation :

$$
C=\left(\frac{\partial \rho}{\partial p}+\frac{\rho}{A} \frac{\partial A}{\partial p}\right)^{-1 / 2}=C_{0}\left(1+c \frac{K D}{e E_{0}}\right)^{-1 / 2}
$$

où $C_{0}=(\partial \rho / \partial p)^{-1 / 2}=\sqrt{K / \rho}$ et $E_{0}$ est le module élastique instantané (Bahrar [9]).

Dans le cas des conduites élastiques, $E_{0}$ correspond au module d'Young. Le coefficient $c$ est un paramètre caractérisant le type d'ancrage de la conduite et qui est fonction du quotient de Poisson $\nu$ (Ghilardi et Paoletti [5]). Dans le cas d'une conduite parfaitement ancrée à ses deux extrémités on aurait une valeur de $c$ telle que :

$c=\left(1-\nu^{2}\right)$ pour les conduites à parois minces.

$c=(1+\nu) * 2 e / D+D\left(1-\nu^{2}\right) /(D+e)$ pour les conduites à parois épaisses.

\section{Résolution par la méthode des caractéristiques}

La méthode des caractéristiques (Fox [13]) est appliquée pour transformer le système d'équations aux dérivées partielles (1) en un système d'équations différentielles ordinaires qui peuvent être intégrées numériquement sans difficulté. On obtient :

$$
\mathrm{d} Q \pm \frac{g S}{C} \mathrm{~d} H+g S J \mathrm{~d} t=0 \text { et } \mathrm{d} x= \pm C \mathrm{~d} t
$$

le + pour les ondes provenant de l'amont et le - pour les ondes provenant de l'aval.

Le coup de bélier est engendré par une discontinuité de fonctionnement (fermeture rapide de vannes). Cette discontinuité se propage et le déplacement est présenté dans le plan $(x, t)$ par les lignes caractéristiques. Dans le cas des conduites quasi rigides telles que les conduites métalliques ou en béton, la vitesse de propagation des ondes de pression $C$ est constante et les lignes caractéristiques sont des droites.

Considérons alors une conduite de longueur $L$ subdivisée en $N$ tronçons égaux de longueur $\Delta x=L / N$. Si on démarre avec les conditions connues du régime permanent à l'instant $t=0$, alors on connaît $Q$ et $H$ aux $N+1$ sections de la conduite. On choisit comme intervalle de temps $\Delta t=\Delta x / C$, et les lignes caractéristiques issues des sections $i-1$ et $i+1$ de la conduite se coupent en une autre section sélectionnée $P$ (Fig. 1).

Dans ces conditions, le maillage régulier du plan $(x, t)$ est identique à celui formé par les lignes caractéristiques et l'intégration des équations (2) permet d'écrire :

$$
\begin{aligned}
& Q_{i}^{k+1}-Q_{i-1}^{k}+\frac{g S}{C}\left(H_{i}^{k+1}-H_{i-1}^{k}\right)+g S J_{i-1}^{k} \Delta t=0 \\
& Q_{i}^{k+1}-Q_{i+1}^{k}-\frac{g S}{C}\left(H_{i}^{k+1}-H_{i+1}^{k}\right)+g S J_{i+1}^{k} \Delta t=0
\end{aligned}
$$

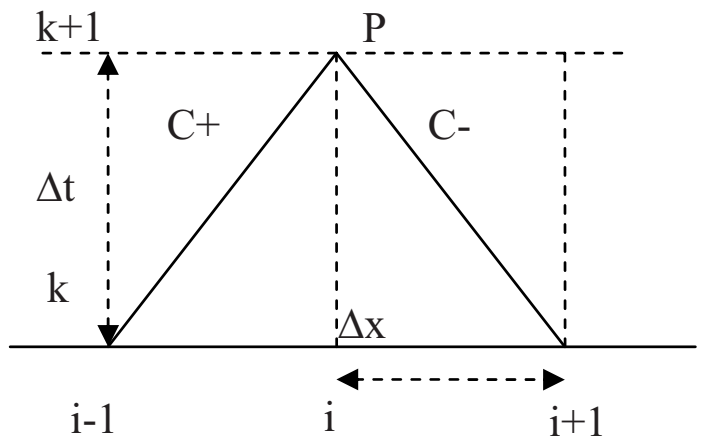

Fig. 1. Lignes caractéristiques : maillage régulier.

où $Q_{i}^{k+1}$ est le débit à l'instant $t+\Delta t$ et $Q_{i-1}^{k}$ est le débit à l'instant $t$.

Le calcul du débit au niveau de chaque section $(i)$ est obtenu en résolvant les équations (3) et (4) pour $Q_{i}^{k+1}$ par élimination de $H_{i}^{k+1}$. Ainsi, on obtient :

$$
\begin{aligned}
Q_{i}^{k+1}=\frac{1}{2}\left[Q_{i-1}^{k}+Q_{i+1}^{k}+\right. & \frac{S g}{C}\left(H_{i-1}^{k}-H_{i+1}^{k}\right) \\
& \left.-S g \Delta t\left(J_{j-1}^{k}+J_{j+1}^{k}\right)\right]
\end{aligned}
$$

et de même pour $H_{i}^{k+1}$ :

$$
\begin{aligned}
H_{i}^{k+1}=\frac{1}{2}\left[H_{i-1}^{k}+H_{i+1}^{k}+\frac{C}{S g}\right. & \left(Q_{i-1}^{k}-Q_{i+1}^{k}\right) \\
& \left.-C \Delta t\left(J_{i-1}^{k}-J_{i+1}^{k}\right)\right]
\end{aligned}
$$

La pression est calculée par la formule :

$$
p_{i}^{k+1}=\rho g\left(H_{i}^{k+1}-Z_{i}\right)
$$

\section{Conditions aux limites}

Les conditions aux limites sont les lois imposées aux variables principales de l'écoulement (le débit $Q$ et la charge $H$ ) aux deux extrémités de la conduite. Ces lois conditionnent généralement le développement et l'évolution des écoulements transitoires.

Au niveau de chaque extrémité d'une conduite unique, seule une des équations caractéristiques (3) ou (4) des deux variables dépendantes $Q_{i}^{k+1}$ et $H_{i}^{k+1}$ est disponible. Une équation auxiliaire explicitant la condition à la limite est donc nécessaire dans chaque cas pour spécifier $Q^{k+1}$ ou $H^{k+1}$. Dans la présente étude seules les conditions aux limites : réservoir à niveau constant, vannes à fermeture instantanée et branchement de conduites, sont considérés.

Considérons donc un nœud $B$ auquel aboutissent $n$ tronçons. Le débit dans chaque tronçon d'indice $i$ est désigné par $Q_{i}$. Le sens conventionnel de circulation des débits est choisi vers le nœud (Fig. 2).

En $B$, l'équation de continuité s'écrit, donc :

$$
Q_{1 B}^{k+1}+Q_{2 B}^{k+1}+Q_{3 B}^{k+1}+\cdots Q_{n B}^{k+1}=0
$$




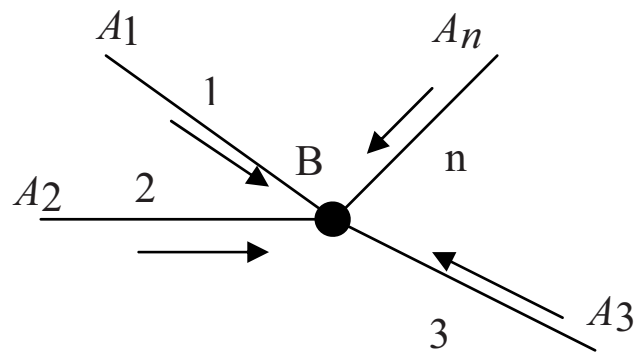

Fig. 2. Jonction de $n$ conduites.

où $Q_{i B}^{k+1}$ est le débit au nœud $i$ à l'instant $(n+1)$.

Sur chaque tronçon, on applique la théorie des caractéristiques.

Pour une onde descendante aboutissant au noud $B$, l'équation (4) donne :

$$
\frac{g S_{i}}{C_{i}} H_{B}^{k+1}+Q_{i B}^{k+1}=\frac{g S_{i}}{C_{i}} H_{A_{i}}^{k}+Q_{i A_{i}}^{k}-\frac{g S_{i}}{C_{i}} J_{i}^{k} L_{i}
$$

Par combinaison des équations (9), en tenant compte de l'équation de continuité (8), il vient :

$$
\begin{aligned}
\left(\sum_{i=1}^{n} \frac{g S_{i}}{C_{i}}\right) H_{B}^{k+1}=\sum_{i=1}^{n} & \left(\frac{g S_{i}}{C_{i}} H_{A_{i}}^{k}\right) \\
& +\sum_{i=1}^{n}\left(Q_{i A_{i}}^{k}\right)-\sum_{i=1}^{n}\left(\frac{g S_{i}}{C_{i}} J_{i}^{k} L_{i}\right)
\end{aligned}
$$

Notons que $J_{i}$ est la valeur pondérée de la perte de charge le long du parcours de l'onde. En l'associant à la forme de pondération suivant la méthode des trapèzes, on obtient :

$$
\int_{A_{i}}^{B P} J \mathrm{~d} x=J_{i}^{k} L_{i}=\frac{J_{i A_{i}}^{k}+J_{i B}^{k+1}}{2} L_{i}
$$

Cette relation permet d'obtenir une valeur approchée de $H_{B}^{k+1}$ à partir d'approximation de $J_{i}^{k}$. Ensuite, on détermine les débits en $B$ dans chaque tronçon par l'équation (8) et par itération, on améliore les valeurs obtenues pour $H_{B}^{k+1}$ et $Q_{i B}^{k+1}$.

On peut aussi envisager de réduire à deux le nombre d'itérations en examinant la résolution d'un système linéarisé.

Nous appliquerons à l'évaluation de $J_{i}^{k}$ la relation suivante (Escande et Thirriot [14]) :

$$
J_{i B}^{k+1} \approx J_{i B}^{k} \frac{2 Q_{i B}^{k+1}-Q_{i B}^{k}}{Q_{i B}^{k}}
$$

Il vient pour la condition de compatibilité (9) le long de la caractéristique montante :

$$
\begin{aligned}
\frac{g S_{i}}{C_{i}} H_{B}^{k+1}+Q_{i B}^{k+1}\left[1+\frac{J_{i B}^{k} g S_{i} L_{i}}{C_{i} Q_{i B}^{k}}\right]= & \\
& \frac{g S_{i}}{C_{i}} H_{A_{i}}^{k}+Q_{i A_{i}}^{k}-\frac{g S_{i}}{C_{i}} L_{i}\left[J_{i A_{i}}^{k}-J_{i B}^{k}\right]
\end{aligned}
$$

où, en posant pour simplifier les écritures $\alpha_{i B}=\frac{g S_{i} L_{i} J_{i B}^{k}}{C_{i} Q_{i B}^{k}}$, on peut écrire le système aux inconnues $H_{B}^{k+1}$ et $Q_{i B}^{k+1}$ sous la forme matricielle suivante :

$$
\begin{aligned}
& {\left[\begin{array}{llccc}
1 & 1 & \cdots & 1 & 0 \\
1+\alpha_{1 B} & 0 & \cdots & 0 & \frac{g S_{1}}{C_{1}} \\
0 & 1+\alpha_{2 B} & \cdots & 0 & \frac{g S_{2}}{C_{2}} \\
\vdots & \vdots & \cdots & \ddots & \vdots \\
0 & 0 & \cdots & 1+\alpha_{n B} & \frac{g S_{n}}{C_{n}}
\end{array}\right]\left\{\begin{array}{l}
Q_{1 B}^{k+1} \\
Q_{2 B}^{k+1} \\
\vdots \\
\vdots \\
Q_{n B}^{k+1} \\
H_{B}^{k+1}
\end{array}\right\}=} \\
&\left\{\begin{array}{l}
0 \\
\frac{g S_{1}}{C_{1}} H_{A_{1}}^{k}+Q_{1 A_{1}}^{k}-\frac{g S_{1}}{C_{1}}\left(J_{1 A_{1}}^{k}-J_{1 B}^{k}\right) \\
\frac{g S_{2}}{C_{2}} H_{A_{2}}^{k}+Q_{2 A_{2}}^{k}-\frac{g S_{2}}{C_{2}}\left(J_{2 A_{2}}^{k}-J_{2 B}^{k}\right) \\
\vdots \\
\frac{g S_{n}}{C_{n}} H_{A_{n}}^{k}+Q_{n A_{n}}^{k}-\frac{g S_{n}}{C_{n}}\left(J_{n A_{n}}^{k}-J_{n B}^{k}\right)
\end{array}\right\}
\end{aligned}
$$

Bien entendu, l'hypothèse où les coefficients $\alpha_{i B}$ ne varient pas de manière sensible au cours de l'écoulement transitoire permet d'inverser la matrice des coefficients en début de calcul. Mais la résolution de ce système linéaire n'est jamais très coûteuse car le nombre de conduites aboutissant en un nœud est très rarement supérieur à quatre.

Les systèmes de conduites peuvent être calculés par la méthode des caractéristiques présentée ci-dessus. On choisit d'abord le pas de temps $\Delta t$. Ensuite, chaque conduite du réseau est subdivisée en $N_{i}=L_{i} /\left(\Delta t C_{i}\right)$ tronçons (nombre entier). Dans ce cas, les variables hydrauliques comprennent deux indices; le premier fait référence au numéro de la conduite et le second indique le numéro de la section. Lorsque $L_{i}-N_{i} \Delta t C_{i} \neq 0$, on applique la méthode d'interpolation linéaire pour intégrer les équations caractéristiques le long des tronçons disproportionnés situés aux extrémités aval des conduites. Ceci nous évite la technique suivie par Samani et Khayatzadeh [3], qui consiste à combiner la méthode des caractéristiques avec la méthode des différences finies lors du calcul de ces tronçons.

Les points intérieurs de toutes les conduites seront calculés, à chaque pas de temps, dans la même procédure. Par contre, les points de connexion des différentes conduites, les extrémités des conduites, les vannes et les branchements, seront tous traités comme des conditions aux limites internes et calculés, à chaque pas de temps, par des procédures différentes. Évidemment, les conditions aux limites du même type seront itérées dans la même procédure.

Dans cette étude, les conditions aux limites suivantes sont considérées :

- Réservoir à niveau constant,

- Fermeture instantanée de vanne,

- Branchement de plusieurs conduites. 
Tableau 1. Conditions initiales.

\begin{tabular}{|c|c|c|c|c|c|c|c|}
\hline $\mathrm{NT}$ & NAM & NAV & $L(\mathrm{~m})$ & $\phi(\mathrm{mm})$ & $Q\left({\mathrm{~L} . \mathrm{s}^{-1}}^{-1}\right)$ & $\mathrm{C}\left(\mathrm{m} \cdot \mathrm{s}^{-1}\right)$ & $\lambda$ \\
\hline 1 & 1 & 2 & 609,6 & 914,400 & 849,500 & 1005,8 & 0,03 \\
\hline 2 & 2 & 3 & 914,4 & 762,000 & 408,7016 & 1143,0 & 0,028 \\
\hline 3 & 2 & 4 & 609,6 & 609,600 & 440,7984 & 1219,2 & 0,024 \\
\hline 4 & 3 & 4 & 548,6 & 457,200 & 176,5611 & 914,4 & 0,02 \\
\hline 5 & 3 & 5 & 457,2 & 457,200 & 232,1405 & 1143,0 & 0,02 \\
\hline 6 & 4 & 5 & 487,7 & 457,200 & 111,1896 & 975,4 & 0,025 \\
\hline 7 & 4 & 6 & 670,6 & 762,000 & 506,1699 & 957,1 & 0,04 \\
\hline 8 & 5 & 6 & 457,2 & 609,600 & 343,3301 & 914,4 & 0,03 \\
\hline 9 & 6 & 7 & 609,6 & 914,400 & 849,500 & 1005,8 & 0,024 \\
\hline & & & Noud & $\mathrm{N}^{\circ}$ (Streeter) & \multicolumn{2}{|c|}{ Charge $H(\mathrm{~m})$} & \\
\hline & & & 1 & Réservoir & \multicolumn{2}{|c|}{191,0000} & \\
\hline & & & 2 & $J_{1}$ & \multicolumn{2}{|c|}{189,2942} & \\
\hline & & & 3 & $J_{2}$ & \multicolumn{2}{|c|}{187,9187} & \\
\hline & & & 4 & $J_{3}$ & \multicolumn{2}{|c|}{186,5040} & \\
\hline & & & 5 & $J_{4}$ & \multicolumn{2}{|c|}{185,8806} & \\
\hline & & & 6 & $J_{5}$ & \multicolumn{2}{|c|}{184,2936} & \\
\hline & & & 7 & Vanne & \multicolumn{2}{|c|}{182,9290} & \\
\hline
\end{tabular}

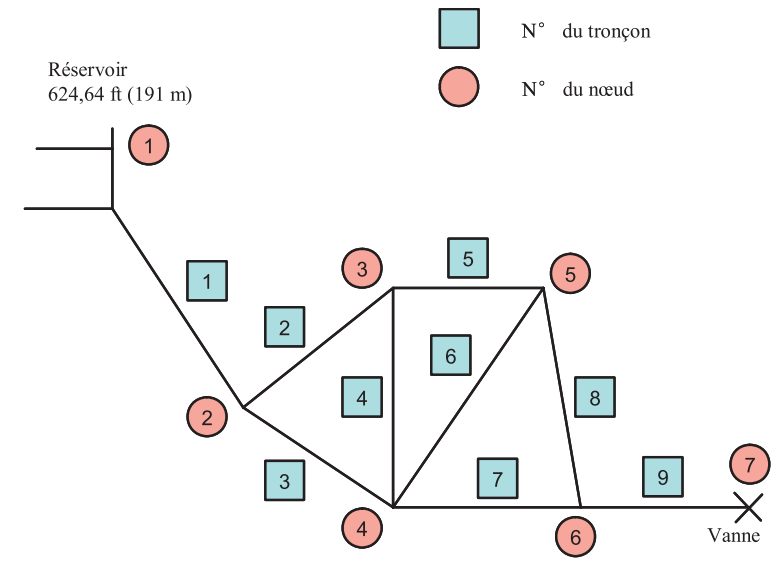

Fig. 3. Réseau maillé de conduites [4].

\section{Applications et résultats}

\subsection{Réseau maillé}

Afin de valider le modèle numérique proposé, nous examinons d'abord le cas de l'écoulement transitoire dans un réseau maillé de conduites schématisé sur la figure 3. L'écoulement transitoire dans ce réseau a été étudié par Streeter et Wylie [4]. Selon les valeurs des célérités qui varient de 914,4 à 1219,2 m.s ${ }^{-1}$, les conduites sont supposées élastiques quasi-rigides (en acier, en fonte ou en béton).

\subsubsection{Conditions initiales (écoulement permanent)}

Les conditions initiales sont établies à l'aide d'un programme de calcul automatique des écoulements permanents par la méthode de Hardy-Cross (Rossi [15]). Ce programme permet le calcul de la répartition des débits dans le réseau et la perte de charge correspondante sur chaque tronçon. Il tient compte de la cote piézométrique qui peut
Tableau 2. Conditions aux limites.

\begin{tabular}{|l|l|}
\hline Nœud & Conditions aux limites \\
\hline 1 (Réservoir à niveau constant) & $H_{1}(t)=191 \mathrm{~m} \forall t$ \\
7 (Vanne à fermeture rapide) & $Q_{7}(t)=0 \forall t$ \\
\hline
\end{tabular}

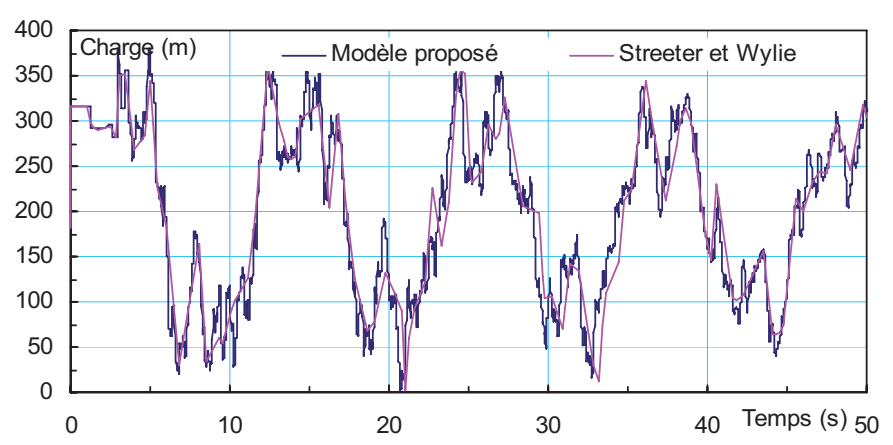

Fig. 4. Fluctuation de la charge au nœud 7 (vanne).

être imposée à certains nœuds du réseau (ici le nœud 1 qui correspond à un réservoir). Les conditions initiales ainsi obtenues sont présentées sur le tableau 1. Ces conditions permettent de démarrer le calcul, par la méthode des caractéristiques, de l'évolution des paramètres de l'écoulement transitoire aux différents nœuds du réseau.

\subsubsection{Conditions aux limites}

Les conditions aux limites aux nœuds sont données dans le tableau 2.

\subsubsection{Validation numérique}

Sur les figures 4 et 5 , nous présentons respectivement la variation, en fonction du temps, de la charge aux nœuds 2 et 7 . Ces résultats concordent parfaitement avec ceux obtenus par Streeter et Wylie [4]. Ces figures mettent 
Tableau 3. Caractéristiques physiques de la conduite 9 .

\begin{tabular}{llll}
\hline Caractéristiques & Conduite en acier & Conduite en PE & Conduite en PVC \\
\hline Diamètre intérieur & $0,9144 \mathrm{~m}$ & $0,9144 \mathrm{~m}$ & $0,9144 \mathrm{~m}$ \\
Épaisseur & $0,0084 \mathrm{~m}$ & $0,09144 \mathrm{~m}$ & $0,09144 \mathrm{~m}$ \\
Longueur & $609,6 \mathrm{~m}$ & $609,6 \mathrm{~m}$ & $609,6 \mathrm{~m}$ \\
Module de Young instantané & $E_{0}=206000 \mathrm{~N} . \mathrm{mm}^{-2}$ & $E_{0}=874 \mathrm{~N}^{-2}$ & $E_{0}=69,6 \mathrm{~N} . \mathrm{mm}^{-2}$ \\
Coefficient de Poisson & 0,27 & $\nu=0,46$ & $\nu=0,5$ \\
Coefficient $c$ & $c=0,9271$ & $c=1,0087$ & $c=0,982$ \\
Célérité & $C=1005,8{\mathrm{~m} . \mathrm{s}^{-1}}^{-1}$ & $C=288,2{\mathrm{~m} . \mathrm{s}^{-1}}^{-1}$ & \\
\hline
\end{tabular}

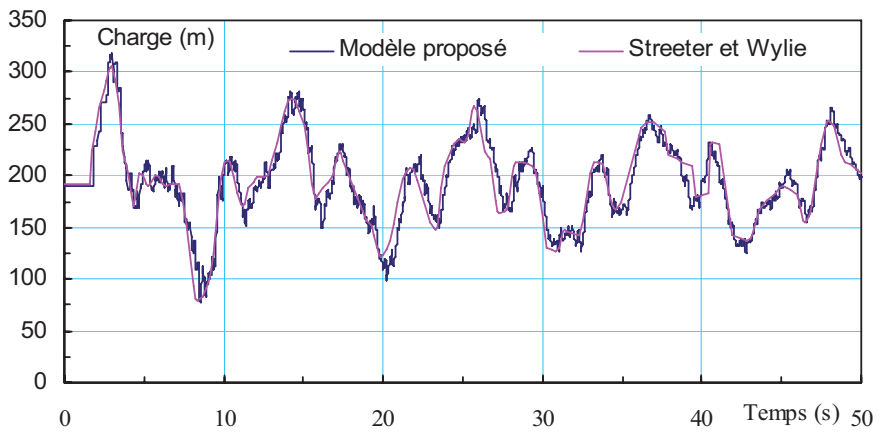

Fig. 5. Fluctuation de la charge au nœud $2\left(J_{1}\right)$.

en évidence la complexité du phénomène de coup de bélier dans les systèmes de conduites à caractéristiques multiples et la difficulté de prévision de la surpression maximale. Contrairement à ce qui est prévu (Jlali et al. [16]), on constate que la surpression maximale (375 $\mathrm{m}$ au niveau de la vanne) ne correspond pas nécessairement au premier maximum de pression obtenu $(317 \mathrm{~m})$. D'ailleurs on peut observer l'apparition de nombreux maxima et la variation de leur amplitude. En poursuivant le calcul jusqu'à un temps de $150 \mathrm{~s}$ (voir Fig. 6), on constate que les pertes d'énergie dans les différents tronçons du réseau écrêteront très sensiblement les amplitudes de ces maxima.

\subsubsection{Influence de l'élasticité des parois}

Afin d'amortir ces surpressions et réduire la durée du phénomène de coup de bélier dans le réseau maillé, nous avons examiné, par simulation numérique, l'influence de l'élasticité de la paroi sur la propagation des ondes de pression en remplaçant la conduite 9, conduite élastique quasi rigide, par une conduite en matériau déformable. Deux types de conduite sont considérés : en polyéthylène (PE) ou en polychlorure de vinyle (PVC), caractérisées par deux comportements viscoélastiques différents. Les caractéristiques physiques de ces conduites sont présentés sur le tableau 3 (Ghilardi et Paoletti [5]).

Les fluctuations de la charge à la vanne (nœud 7) et à la jonction $J_{1}$ (nœud 2) sont portées sur les figures 6 et 7 pour les trois types de conduites utilisées pour le tronçon 9 (quasi-rigide : $C=1005,8 \mathrm{~m} . \mathrm{s}^{-1}$, en PE : $C=$ $288,2 \mathrm{~m} . \mathrm{s}^{-1}$ et en PVC : $C=84,2 \mathrm{~m} . \mathrm{s}^{-1}$ ).

Ces résultats montrent clairement que le fait de remplacer une conduite quasi-rigide par une conduite

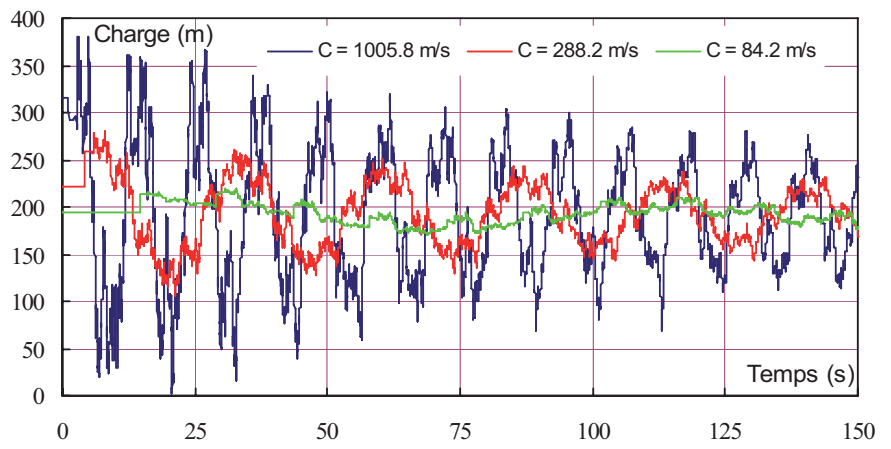

Fig. 6. Évolution de la charge au noud 7 (vanne).

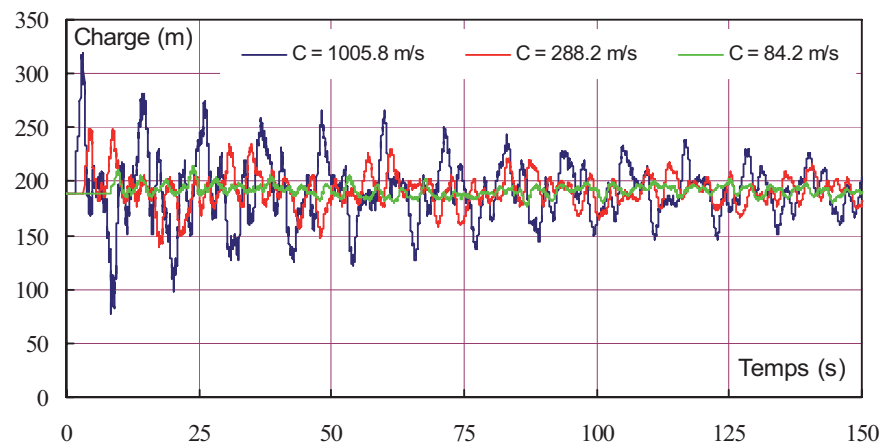

Fig. 7. Évolution de la charge au nœud $2\left(J_{1}\right)$.

élastique de grande déformabilité constitue un système utile pour atténuer les surpressions.

On note, en effet, que la présence de la conduite élastique atténue la première surpression qui atteint, à la vanne, la valeur 221,6 $\mathrm{m}$ pour la conduite en $\mathrm{PE}$ et $194,7 \mathrm{~m}$ pour la conduite en PVC au lieu de $316,2 \mathrm{~m}$ pour la conduite quasi-rigide.

Les surélévations de pression qui succèdent montrent que les conduites élastiques réduisent le régime transitoire dû à l'étalement du front d'onde causé par le facteur simultané à savoir la petite célérité dans la conduite élastique, qui segmente le coup de bélier en plusieurs ondes élémentaires.

On constate, aussi, que la réduction de la surpression est plus importante avec la conduite en PVC qu'avec la conduite en polyéthylène. 
Tableau 4. Caractéristiques du réseau du Sud Tunisien (conditions initiales).

\begin{tabular}{|c|c|c|c|c|c|c|}
\hline Tronçon & \multicolumn{2}{|c|}{ Nœuds } & \multirow{2}{*}{$\begin{array}{l}\text { longueur }(\mathrm{m}) \\
6630,0\end{array}$} & \multirow{2}{*}{$\frac{\text { Diamètre }(\mathrm{mm})}{800.0}$} & \multirow{2}{*}{$\begin{array}{c}\text { Débit }\left(1 . \mathrm{s}^{-1}\right) \\
218,00\end{array}$} & \multirow{2}{*}{$\begin{array}{c}\text { Célérité }\left({\left.\mathrm{m} . \mathrm{s}^{-1}\right)}_{1000,0}\right. \\
\end{array}$} \\
\hline 1 & 1 & 2 & & & & \\
\hline 2 & 2 & 3 & 1615,0 & 600,0 & 218,00 & 1000,0 \\
\hline 3 & 3 & 4 & 8370,0 & 600,0 & 218,00 & 1000,0 \\
\hline 4 & 4 & 5 & 5000,0 & 600,0 & 218,00 & 1000,0 \\
\hline 5 & 5 & 6 & 8570,0 & 600,0 & 218,00 & 1000,0 \\
\hline 6 & 6 & 7 & 6635,0 & 600,0 & 218,00 & 1000,0 \\
\hline 7 & 7 & 8 & 7043,0 & 600,0 & 158,00 & 1000,0 \\
\hline 8 & 8 & 9 & 7043,0 & 600,0 & 158,00 & 1000,0 \\
\hline 9 & 9 & 10 & 7042,0 & 600,0 & 158,00 & 1000,0 \\
\hline 10 & 10 & 11 & 6643,0 & 500,0 & 158,00 & 1000,0 \\
\hline 11 & 11 & 12 & 7690,0 & 500,0 & 158,00 & 1000,0 \\
\hline 12 & 7 & 13 & 4500.0 & 450,0 & 60,00 & 1000,0 \\
\hline 13 & 13 & 14 & 3500,0 & 450,0 & 60,00 & 1000,0 \\
\hline 14 & 14 & 15 & 6450,0 & 450,0 & 60,00 & 1000,0 \\
\hline 15 & 15 & 16 & 4650,0 & 450,0 & 60,00 & 1000,0 \\
\hline 16 & 16 & 17 & 4650,0 & 450,0 & 60,00 & 1000,0 \\
\hline & & & Nœud & Cote au sol (m) & Niveau piezo. (m) & \\
\hline & & & 1 & 102,300 & 102,300 & \\
\hline & & & 2 & 68,000 & 100,180 & \\
\hline & & & 3 & 65,500 & 99,190 & \\
\hline & & & 4 & 38,000 & 92,590 & \\
\hline & & & 5 & 27,000 & 88,650 & \\
\hline & & & 6 & 18,000 & 81,900 & \\
\hline & & & 7 & 7,000 & 76,670 & \\
\hline & & & 8 & 5,000 & 73,610 & \\
\hline & & & 9 & 5,000 & 70,550 & \\
\hline & & & 10 & 1,500 & 67,500 & \\
\hline & & & 11 & 1,000 & 60,320 & \\
\hline & & & 12 & 47,000 & 52,000 & \\
\hline & & & 13 & 7,000 & 73,890 & \\
\hline & & & 14 & 10,000 & 71,730 & \\
\hline & & & 15 & 15,000 & 67,730 & \\
\hline & & & 16 & 15,000 & 64,860 & \\
\hline & & & 17 & 58,500 & 62,000 & \\
\hline
\end{tabular}

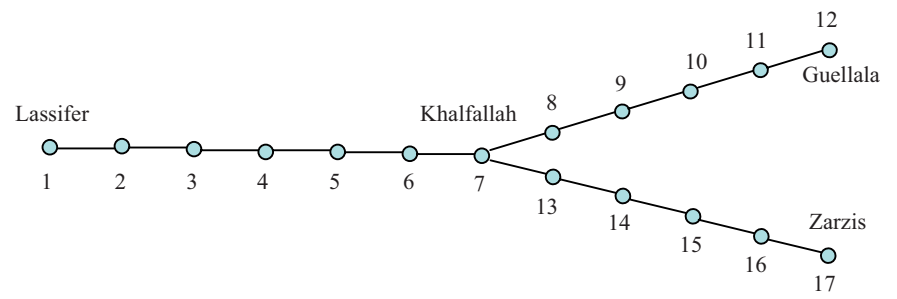

Fig. 8. Réseau du Sud Tunisien.

\subsection{Réseau ramifié : Réseau d'adduction en eau du Sud Tunisien}

\subsubsection{Description du système et position du problème}

La figure 8 présente le système en $Y$ de liaison entre le réservoir amont de Lassifer et les réservoirs aval de Guellala, dans l'île de Djerba, et de Zarzis. Les données de ce réseau ont été fournies par la Société Nationale d'Exploitation et de Distribution des Eaux en Tunisie (SONEDE). Les conditions du régime permanent initial sont indiquées sur le tableau 4.

\subsubsection{Fermeture instantanée à Guellala}

Toutes les conduites qui sont en fonte ont une pression d'épreuve maximale égale à $1,5 \mathrm{MPa}$. Ce réseau est alimenté par un réservoir à niveau constant situé au nœud 1 . Il comprend également deux réservoirs à niveau constant aux nœuds 12 et 17 équipés d'obturateurs (vannes à fermeture instantanée).

Les causes d'incident sont dues à une fermeture intempestive de la vanne à l'extrémité de l'antenne de Guellala (fermeture qu'on supposera quasi instantanée au nœud 12).

\subsubsection{Conduite 11 quasi rigide}

Les résultats sont présentés sur la figure 9. Les calculs ont été réalisés avec une valeur constante de la célérité égale à $1000 \mathrm{~m} \cdot \mathrm{s}^{-1}$. Pour la ligne Lassifer Khalfallah et Zarzis, on peut signaler que l'évolution du régime transitoire reste partout acceptable. Par contre, pour la branche Khalfallah-Guellala, il y a une légère surpression et notamment au voisinage du nœud 12. Les calculs confirment les résultats obtenus par l'algorithme simplifié 

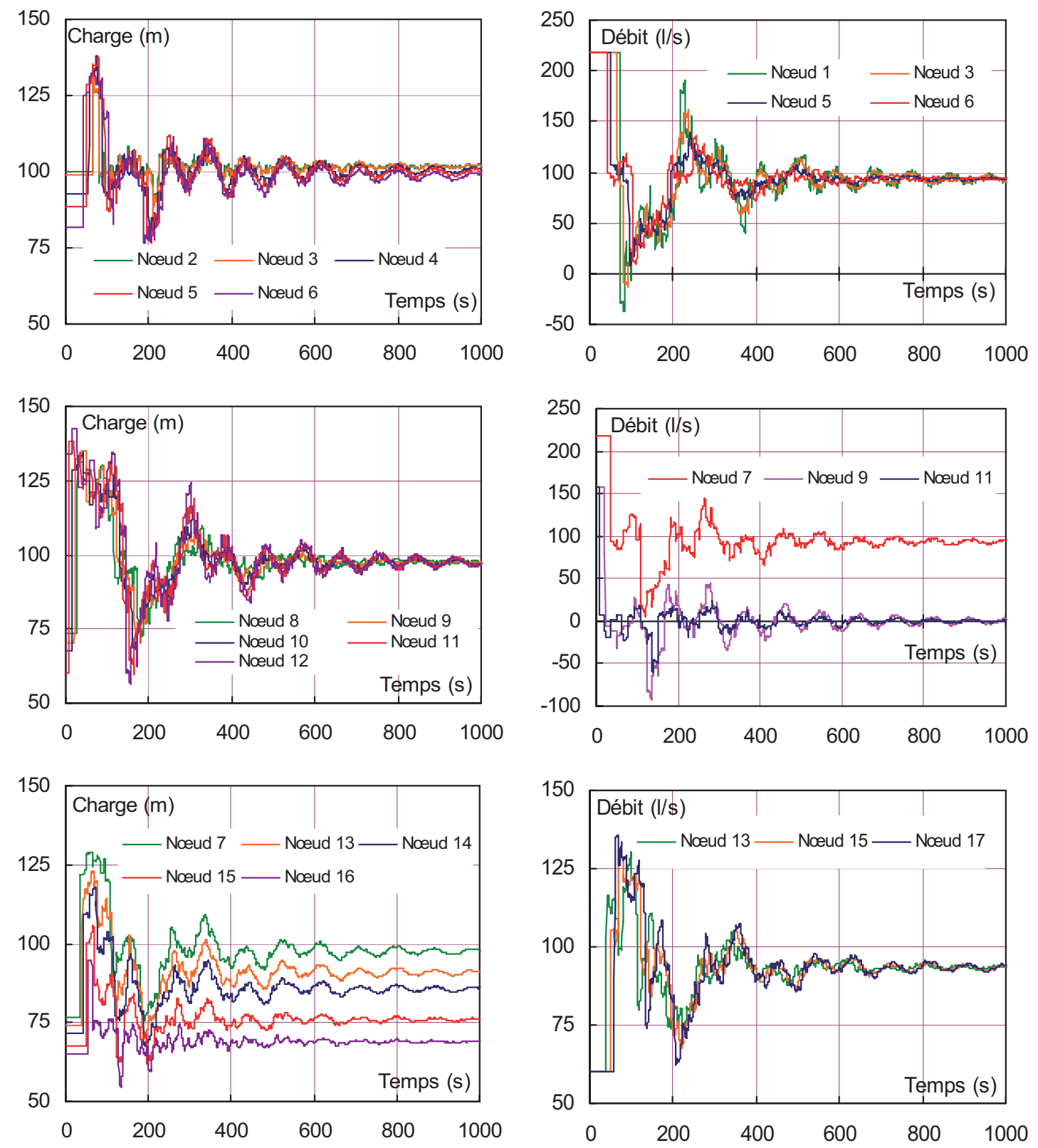

Conduite 11 en acier, $C=1000 \mathrm{~m} \cdot \mathrm{s}^{-1}$

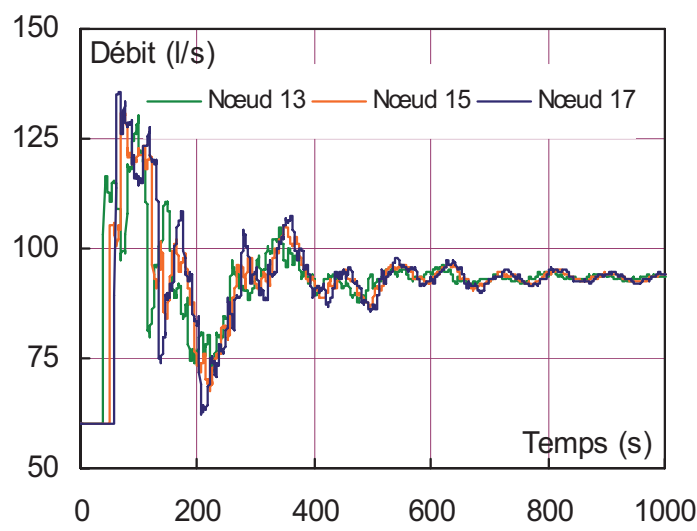

Conduite 11 en acier, $C=1000 \mathrm{~m} \cdot \mathrm{s}^{-1}$

Fig. 9. Évolution de la charge et du débit due à une fermeture brusque à Guellala (nœud 12).

(Jlali et al. [16]). En effet la charge au nœud 12 augmente brusquement de $52 \mathrm{~m}$ à $134 \mathrm{~m}$ qui correspond exactement à la valeur obtenue par l'algorithme simplifié. Mais, du fait des pertes de charge et de la réflexion de l'onde de coup de bélier au noud 11, elle subit, à l'instant 15,5 s, une autre augmentation et passe de 134 à $142 \mathrm{~m}$.

Sur cette même branche (Khalfallah-Guellala), on constate que le débit tend à s'annuler, puisqu'il a été coupé au noud 12, et que les charges convergent vers la charge du noud 7.

Notons aussi que le débit de la branche KhalfallahZarzis augmente et passe de $601 . \mathrm{s}^{-1}$ à environ $95 \mathrm{l} . \mathrm{s}^{-1}$. Pour ce qui est de la branche Lassifer-Khalfallah, il y a diminution du débit de $2181 . \mathrm{s}^{-1}$ à $951 . \mathrm{s}^{-1}$ et donc une augmentation de la charge par rapport à celle du régime permanent initial.

\subsubsection{Conduite 11 élastique}

Sur la figure 10, on présente l'évolution de la charge aux différents nouds du réseau après avoir remplacé la conduite 11-12 quasi-rigide par une conduite déformable. Les mêmes types de conduite sont considérés : en polyéthylène $(\mathrm{PE})$ ou en polychlorure de vinyle (PVC) (voir Tab. 5). Là encore, on constate une atténuation de la surpression transitoire par la présence de la conduite élastique liée à la réduction des vitesses de propagation. Notons que cette atténuation est oscillatoire dans le cas 

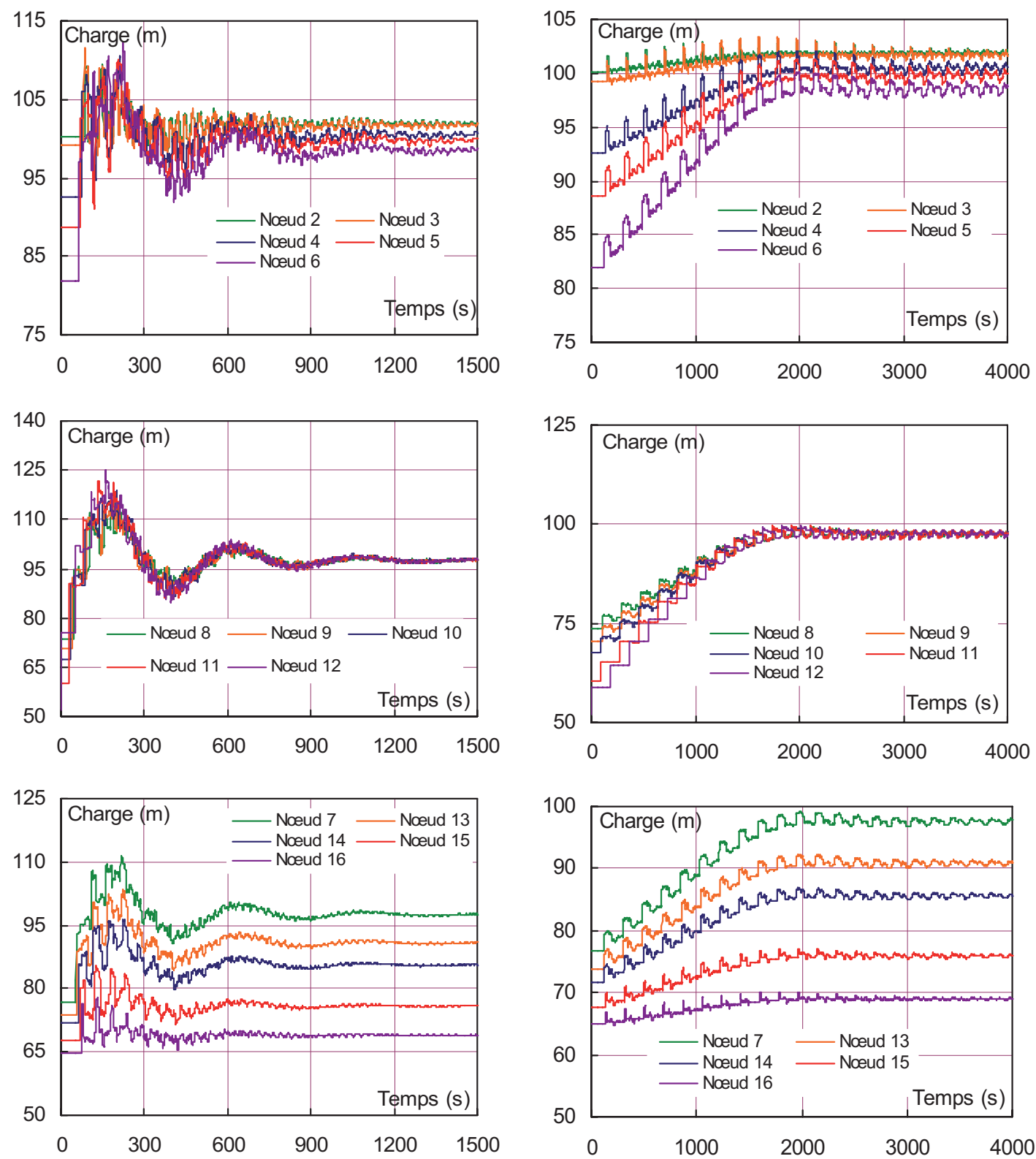

Conduite 11 en polyéthylène, $\mathrm{C}=288.2 \mathrm{~m} / \mathrm{s}$

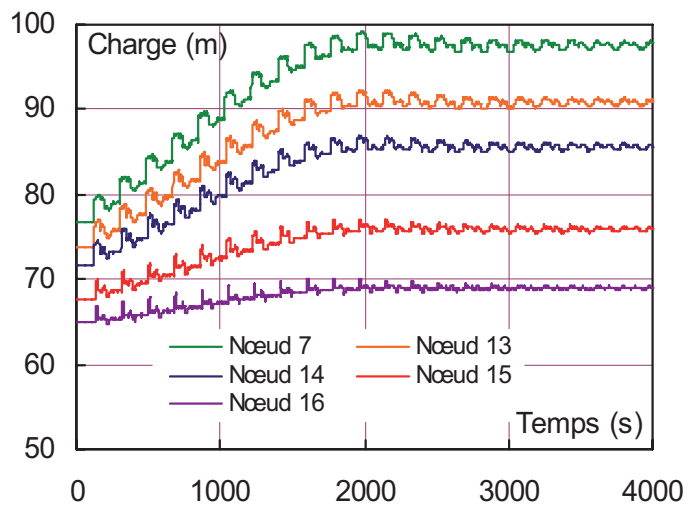

Conduite 11 en PVC, $\mathrm{C}=84.2 \mathrm{~m} / \mathrm{s}$

Fig. 10. Évolution de la charge due à une fermeture brusque à Guellala (nœud 12).

de la conduite en PE et fortement amortie dans le cas de la conduite en PVC. D'autre part, le pas de temps de calcul étant pris égal à $0,25 \mathrm{~s}$, chaque courbe comprend 6000 points pour la conduite en $\mathrm{PE}$ et 16000 points pour la conduite en PVC.

Par ailleurs, la charge au noud 12 augmente brusquement de 52 à $75,6 \mathrm{~m}$ avec la conduite en $\mathrm{PE}$ et de 52 à 52,9 $\mathrm{m}$ pour la conduite en PVC. Par conséquent, la réduction de la surpression est plus importante avec la conduite en PVC qu'avec celle en polyéthylène.

\section{Conclusion}

Dans l'idée de rechercher un dispositif susceptible d'atténuer la propagation des ondes de coup de bélier dans les réseaux de conduites, nous avons développé une modélisation unidimensionnelle et un programme de calcul basé sur la méthode des caractéristiques. À l'aide de ce programme, nous avons étudié d'une part la propagation des ondes de pression le long du système de conduites et d'autre part l'effet d'amortissement d'un tronçon élastique inséré dans le réseau à la place d'un autre tronçon quasi-rigide. Dans ce dernier cas, d'après les résultats, il ressort que si l'on met un tronçon élastique pour protéger un système de conduites, la surpression provoquée par le phénomène de coup de bélier s'amortit très rapidement. Ceci s'explique : tant que le tronçon élastique se dilate, il absorbe la surpression par déformation de la canalisation et l'amortissement est d'autant plus important que l'élasticité de ce tronçon est plus grande. 
Tableau 5. Caractéristiques physiques de la conduite 11.

\begin{tabular}{llll}
\hline Caractéristiques & Conduite en acier & Conduite en PE & Conduite en PVC \\
\hline Diamètre intérieur & $0,5 \mathrm{~m}$ & $0,5 \mathrm{~m}$ & $0,5 \mathrm{~m}$ \\
Épaisseur & $0,0045 \mathrm{~m}$ & $0,05 \mathrm{~m}$ & $0,05 \mathrm{~m}$ \\
Longueur & $7690 \mathrm{~m}$ & $7690 \mathrm{~m}$ & $7690 \mathrm{~m}$ \\
Module de YOUNG instantané & $E_{0}=206000 \mathrm{~N} \cdot \mathrm{mm}^{-2}$ & $E_{0}=874 \mathrm{~N}^{-2}$ & $E_{0}=69,8 \mathrm{Nm}^{-2} \mathrm{~mm}^{-2}$ \\
Coefficient de Poisson & 0,27 & $\nu=0,46$ & $\nu=0,5$ \\
Coefficient $c$ & $c=0,9271$ & $c=1,0087$ & $c=0,982$ \\
Célérité & $C=1000{\mathrm{~m} . \mathrm{s}^{-1}}^{-1}$ & $C=288,2{\mathrm{~m} . \mathrm{s}^{-1}}^{-1}$ & \\
\hline
\end{tabular}

Bien que les résultats présentés dans cette étude sont loin d'avoir un caractère exhaustif, nous avons pu mettre en évidence le rôle de l'élasticité de la paroi qui intervient comme un mécanisme dissipatif contribuant de façon notable à l'amortissement des perturbations.

Cependant, beaucoup de questions restent encore sans réponse satisfaisante et ne peuvent être résolues que si l'on tient compte de la loi générale du comportement viscoélastique du tronçon inséré.

\section{Références}

[1] W. Mpesha, M.H. Chaudry, S.L. Gassman, Leak detection in pipes by frequency response method using a step excitation, J. Hydraulic Res. 40 (2000) 55-62

[2] P.F. Boulos, D.J. Wood, J.E. Funk, Pipe network transient-distributed and lumped parameter modeling, 6th International Conference on Pressure Surges, Cambridge, England, 4-6 October 1989

[3] H.M.V. Samani, A. Khayatzadeh, Transient flow in pipe networks, J. Hydraulic Res. 40 (2002) 637-644

[4] V.L. Streeter, E.B. Wylie, Hydraulic transients, F.E.B. Press, Ann Arbor, 1983

[5] P. Ghilardi, A. Paoletti, Additionnal viscoelastic pipes as pressure surges suppressors, 5th Int. Conf. Pressure Surges, 22-24 September 1986, pp. 113-121

[6] M. S. Güney, Waterhammer in viscoelastic pipes where cross-section parameters are time dependent, 4th Int. Conf. Pressure Surges, 1983, pp. 189-204
[7] M. Gally, M.S. Güney, E. Rieutord, An investigation of pressure transients in viscoelastic pipes, J. Fluids Eng. 101 (1979) 495-499

[8] L. Suo, E.B. Wylie, Complex wave speed and hydraulic transients in viscoelastic pipes, J. Fluids Eng. 112 (1990) 496-500

[9] B. Bahrar, Influence sur les écoulements transitoires en conduites, des termes d'inertie de la paroi, ainsi que des déformations de flexion et de cisaillement, Thèse de Doctorat $3^{\mathrm{e}}$ cycle, INSA de Lyon, 1986

[10] P. Germain, Mécanique, École Polytechnique, Ellipses, 1986

[11] M. Carlier, Hydraulique générale et appliquée, Édition Eyrolles-Paris, 1972

[12] S. Stuckenbruck, D.C. Wiggert, R.S. Otwell R., The influence of pipe motion on acoustic wave propagation, J. Fluids Eng. 107 (1985) 518-522

[13] J.A. Fox, Hydraulic analysis of unsteady flow in pipe networks, The Mac Millan Press LTD., London, 1977

[14] L. Escande, C. Thirriot, Propagation des fronts de surpressions dues à des fermetures de vannes dans le réseau ramifié du Bas Léon, Rapport LE 271 et CT 117, IMF Toulouse, 1968

[15] G. Rossi, Interpolation de la formule de Colebrook pour l'application de la méthode de Hardy Cross aux réseaux de distribution d'eau, La Houille Blanche 8 (1984) 553556

[16] A. Jlali, E. Hadj-Taïeb, C. Thirriot, Algorithme simplifié de calcul de phénomènes de propagation d'ondes dans un système de conduites avec embranchement, La Houille Blanche, accepté, juillet 2004 